\title{
TRÊS MESES DE TREINAMENTO FÍSICO MELHORA A COMPOSIÇÃO CORPORAL E APTIDÃO FÍSICA DE BOMBEIROS
}

\section{Three months of physical training improves fireman's body composition and fitness}

\author{
Marcio Robson Verzola'; George Vieira²; Edio Luiz Petroski \\ ${ }^{1}$ Corpo de Bombeiros Militar do Estado de Santa Catarina (CBMSC) - Florianópolis - Santa Catarina - Brasil. \\ ${ }^{2}$ Laboratório de Esforço Físico (LAEF) - Universidade Federal de Santa Catarina - Florianópolis - Santa Catarina - Brasil. \\ ${ }^{3}$ Núcleo de Cineantropometria e Desempenho Humano (NUCIDH) - Universidade Federal de Santa Catarina - Florianópolis \\ Santa Catarina - Brasil.
}

\begin{abstract}
Resumo: Os componentes da aptidão física de militares tem sido foco de atenção na comunidade científica haja vista sua repercussão positiva no desempenho físico e mental durante a realização de atividades profissionais. O estudo teve como objetivo, verificar os efeitos de 12 semanas treinamento físico sobre a composição corporal e aptidão física de bombeiros militares de Santa Catarina. Foram avaliados 52 alunos saudáveis e aptos para iniciar o curso de formação. Todas as avaliações foram realizadas antes e após o programa de treinamento. Na avaliação antropométrica foi determinado o percentual de gordura, massa magra (MM) e massa de gordura (MG). A aptidão física foi avaliada utilizando-se de um protocolo de avaliação contida no manual C-2020 do Exército Brasileiro, composto de flexão na barra fixa, abdominal, 100m velocidade e corrida 3200m. Por meio do tempo obtido no teste de $3200 \mathrm{~m}$ foi estimado o consumo máximo de oxigênio (VO2max). 0 treinamento físico teve duração de 12 semanas, com três sessões semanais, de aproximadamente 90 minutos. Foram utilizados os testes t de student para amostras pareadas, ou o teste de Wilcoxon, assumindo um nível de significância de $5 \%$. As variáveis antropométricas: IMC, MM e MG modificaram significativamente em média $2,1 \%, 1,4 \%$ e $4,1 \%$ após o treinamento, respectivamente. Na aptidão física ocorreram melhoras significativas de $12 \%, 4,1 \%$ e $4,8 \%$ no desempenho dos Testes 100m, 3200m e VO2max, respectivamente. Além disso, o número de execuções aumentou em média 43,6\% e 31,5\% na barra fixa e abdominal, respectivamente. Conclui-se que o treinamento físico realizado por um período de três meses, parece induzir efeitos positivos na aptidão física e na composição corporal de militares durante o período de formação.
\end{abstract}

Palavras chave: Bombeiros, aptidão física, treinamento físico, composição corporal.

Abstract: The components of physical fitness in the military have been the focus of scientific community attention due to their positive impact on physical and mental performance during professional activities. This study aimed to evaluate the effects of 12 weeks physical training on body composition and physical fitness of firefighters from Santa Catarina. Fifty two male soldiers, students, begun the physical training protocol. All evaluations were performed before and after training. Anthropometric assessment was based on four skinfolds to determine the percentage of body fat (\%F), fat-free mass (FFM) and fat mass (FM). Physical fitness was assessed using a protocol prescribed in the Brazilian Army Field Manual C-2020, consisting of: pull-ups, curl-ups, 100m speed run and 3200m run. Maximal oxygen uptake (VO2max) was estimated by the performance in the $3200 \mathrm{~m}$ test. Physical training lasted 12 weeks, with three weekly sessions of approximately 90 minutes. Statistically, we used the Student $t$ test for paired samples or Wilcoxon test, assuming a significance level of $5 \%$. The anthropometric variables: body mass index, FFM and FM changed significantly in average $2.1 \%, 1.4 \%$ and $4.1 \%$ after training, respectively. Physical fitness was significant improvement of $12 \%$, $4.1 \%$ and $4.8 \%$ in the performance of $100 \mathrm{~m}, 3200 \mathrm{~m}$ and VO2max tests, respectively. Moreover, the number of executions improved on average $43.6 \%$ and $31.5 \%$ in the pull-ups and curl-ups, respectively. We concluded that physical training carried out for a period of three months, seems to induce higher changes in physical tests than on the actual body composition.

Key words: Firefighters, physical fitness, physical training, body composition.

Aceito em 10/09/2009 - Rev. Educ. Fís. 2009 - 11-18. Rio de Janeiro - RJ - Brasil

\section{INTRODUÇÃO}

OCorpo de Bombeiros Militar do Estado de Santa Catarina (CBMSC) têm missões constitucionais de proteção à sociedade e de seus bens, ações de defesa civil, salvamentos diversos e combate a incêndios ${ }^{(1)}$. $\mathrm{Na}$ atividade profissional do bombeiro militar são executadas atividades físicas como: transposição de obstáculos, deslocamentos rápidos em curtas, médias e longas distâncias, transporte de pessoas ou materiais em jornadas de trabalho de 24 horas, tomando decisões importantes em situações de estresse mental e físico.

Desse modo níveis baixo de aptidão física, 
aumentam a propensão ao aparecimento de problemas articulares, posturais e lesões musculares. Estes são problemas freqüentes em militares, os quais não os sentem apenas quando precisam ficar por algumas horas em forma, mas também no seu trabalho diário ${ }^{(2)}$. Particularmente, no caso de bombeiros, Rhea et al., (3) demonstraram que a performance no trabalho pode estar associada a níveis de força e resistência muscular numa amplitude maior que o próprio nível de aptidão aeróbia.

Neste sentido, existem evidências mostrando que militares com bom condicionamento aeróbio melhoram seu desempenho na função exercida além de minimizar os efeitos do estresse na tomada de decisões ${ }^{(4)}$.

Deve-se ressaltar que, o conhecimento do nível de aptidão física dos militares de uma determinada corporação torna-se fundamental para a tomada de decisão de um comandante sobre como empregar estes militares. Conseqüentemente, a aptidão física dos militares deve ser desenvolvida e avaliada sistematicamente ${ }^{(5)}$.

A melhoria da aptidão física contribui para o aumento significativo da prontidão dos militares para a missão. Além disso, vale ressaltar que militares bem preparados fisicamente têm mais condições de suportar o estresse extremo da função militar ${ }^{(6)}$.

Neste sentido o emprego do teste de aptidão física (TAF) constitui uma importante ferramenta de seleção e classificação básica dentro de um processo de avaliação ${ }^{(7)}$, bem como um instrumento fundamental para o controle e monitoramento dos efeitos de um programa de treinamento sobre a composição corporal, a qual é fortemente associada à saúde, à aptidão física e ao desempenho nas tarefas laborais.

O serviço militar requer uma constante renovação do corpo de funcionários, isso requer a formação de novos integrantes, os quais são submetidos a períodos de treinamento específico. Tal cuidado com a preparação objetiva entre outros aspectos, melhorar a condição física do militar proporcionando melhores condições de cumprir com suas atribuições funcionais e sua saúde.

Estudos recentes ${ }^{(8,9)}$ envolvendo oito semanas e dez meses treinamento, têm comprovado efeitos positivos do treinamento físico sobre a composição corporal e os componentes da aptidão física de militares do exército e de oficiais da reserva.
Apesar das evidências encontradas na literatura, a variabilidade de métodos de treinamento físico empregados nas pesquisas, as diferenças de características das amostras estudadas, assim como os diferentes períodos de treinamento investigados, parecem justificar a realização de novos estudos buscando melhor entender as situações cada vez mais específicas, particularmente no que diz respeito à preparação de militares do corpo de bombeiros.

Face ao exposto, o objetivo deste estudo foi verificar o efeito de 12 semanas treinamento físico sobre a composição corporal e aptidão física de Bombeiros Militares do Estado de Santa Catarina.

\section{MATERIAIS E MÉTODOS}

\section{Sujeitos}

A seleção da amostra foi realizada de modo intencional não probabilística, sendo composta por 52 alunos, do sexo masculino com média de idade de 21,62 $\pm 2,21$ anos e estatura 1,78 $\pm 0,05 \mathrm{~m}$, todos integrantes do curso de formação de soldados do Corpo de Bombeiros Militar do Estado de Santa Catarina. Como critério de inclusão os sujeitos teriam de ser considerados aptos pela uma Junta de Inspeção de Saúde composta pela Organização do Corpo de Bombeiros Militar. Os exames analisados foram os seguintes: exames médicos (hemograma completo; glicemia de jejum; creatinina sérica; sorologia para Lues - VDRL quantitativo; parcial de urina; radiografia de tórax PA com laudo; radiografia de coluna cérvico-tóraco-lombo-sacra com laudo; e eletrocardiograma).

\section{Desenho do estudo}

Previamente ao período de três meses de treinamento físico os sujeitos foram submetidos a dois dias de coleta de dados. No primeiro dia foi realizada a avaliação antropométrica, e no dia seguinte os avaliados realizaram os testes correspondentes ao TAF como preconiza o manual C20-20. Após, 48 horas de descanso iniciou o programa de exercícios, e na semana seguinte, ao término do treinamento foram realizadas as mesmas coletas de dados do período pré-treinamento.

\section{Prescrição do treinamento físico}

Com base no teste de aptidão física foi prescrito o programa de exercícios de acordo com as recomendações do ACSM ${ }^{(10)}$, como descrito no QUADRO 1. 


\section{QUADRO 1}

Planejamento do treinamento realizado ao longo de 12 semanas.

$1^{\text {a }}$ a $7^{\text {a }}$ semana treinamento aeróbio.

\begin{tabular}{|c|c|c|c|c|}
\hline Segunda & Terça & Quarta & Quinta & Sexta \\
\hline $\begin{array}{l}\text { Aq. }+ \text { along. }=15 \mathrm{~min} \\
\quad \mathrm{TCP}=60 \mathrm{~min} \\
\mathrm{VC}+\text { along. }=15 \mathrm{~min}\end{array}$ & Folga & $\begin{array}{l}\text { Aq. }+ \text { along. }=15 \mathrm{~min} \\
\quad \mathrm{TCP}=60 \mathrm{~min} \\
\mathrm{VC}+\text { along. }=15 \mathrm{~min}\end{array}$ & Folga & $\begin{array}{l}\text { Aq. }+ \text { along. }=15 \mathrm{~min} \\
\quad \mathrm{TCP}=60 \mathrm{~min} \\
\mathrm{VC}+\text { along. }=15 \mathrm{~min}\end{array}$ \\
\hline \multicolumn{5}{|c|}{$5^{a}$ a $8^{a}$ semana inclusão de treinos de força } \\
\hline $\begin{array}{l}\text { Aq. }+ \text { along. }=15 \mathrm{~min} \\
\mathrm{TCP}+\mathrm{TNM}=45 \mathrm{~min} \\
\mathrm{VC}+\text { along }=15 \mathrm{~min} .\end{array}$ & Folga & $\begin{array}{l}\text { Aq. }+ \text { along. }=15 \mathrm{~min} \\
\text { TCP }+ \text { TNM }=45 \mathrm{~min} \\
V C+\text { along }=15 \mathrm{~min} .\end{array}$ & Folga & $\begin{array}{l}\text { Aq. }+ \text { along. }=15 \mathrm{~min} \\
\mathrm{TCP}+\mathrm{TNM}=45 \mathrm{~min} \\
\mathrm{VC}+\text { along }=15 \mathrm{~min} .\end{array}$ \\
\hline \multicolumn{5}{|c|}{$5^{\mathrm{a}}$ a $8^{\mathrm{a}}$ semana inclusão de treinos de força } \\
\hline $\begin{array}{l}\text { Aq. }+ \text { along. }=15 \mathrm{~min} \\
\text { TCP }=20 \mathrm{~min} \\
\text { TNM }=20 \mathrm{~min} . \\
\mathrm{VC}+\text { along }=15 \mathrm{~min} .\end{array}$ & Folga & $\begin{array}{l}\text { Aq. }+ \text { along. }=15 \mathrm{~min} \\
\mathrm{TCP}+\mathrm{TNM}=45 \mathrm{~min} \\
\mathrm{VC}+\text { along }=15 \mathrm{~min} .\end{array}$ & Folga & $\begin{array}{c}\text { Aq. + along. = } 15 \mathrm{~min} \\
\text { TCP }=30 \mathrm{~min} \\
\text { TNM }=20 \mathrm{~min} . \\
\mathrm{VC}+\text { along }=15 \mathrm{~min} .\end{array}$ \\
\hline
\end{tabular}
Aq. $=$ aquecimento; along. $=$ alongamento; $\mathrm{TCP}=$ Trabalho Cardiopulmonar; $\mathrm{TNM}=$ Trabalho neuromuscular. $\mathrm{VC}=$ volta à calma;

O controle da intensidade foi realizado por meio da percepção subjetiva de esforço (PSE) (escala de Borg 6 -20) devido heterogeneidade do grupo avaliado (11). Os avaliados foram familiarizados com a escala e orientados a realizarem os testes contínuos em valores de PSE entre 15 (intenso - pesado) e 17 (muito intenso).

Ao longo deste período de treinamento o grupo realizou sessões de treinamento aeróbico e neuromuscular, sendo empregado predominantemente o método de treinamento contínuo. Como preconizado pelo ACSM (10) para aprimorar a capacidade cardiopulmonar, foram realizadas também sessões de treinamento intervalado com intensidades mais elevadas (PSE próxima ao valor 19 - extremamente intenso) visando aprimorar a potência aeróbica, e ainda sessões de "circuit training" contendo exercícios localizados englobando todos os grupos musculares (TNM).

Com o intuito de promover variações nos estímulos de treinamento, mantendo assim o grupo motivado e provocando novas adaptações, foram executadas 8 sessões de treinamento realizando performances máximas nas distâncias de 200 m, 400m, 600m 800m e 1000m (potência) e ainda 4 sessões de treinamento baseado no método "Fartlek" (TNM + TCP). O treinamento de força ou resistência muscular localizada foi composto por sessões contemplando exercícios de flexões no solo, abdominais, flexões na barra fixa, circuitos com pesos auxiliares e com o próprio peso dos participantes.

$\mathrm{Na}$ maioria das sessões os trabalhos foram conduzidos de forma combinada entre os métodos cardiopulmonar e resistência, isso justifica o número total de trabalho cardiopulmonar ser mais elevado do que o treinamento neuromuscular.

\section{Avaliação da composição corporal}

A massa corporal (MC) foi medida em uma balança digital devidamente aferida e que apresentava precisão de $0,1 \mathrm{~kg}$ (Filizola, São Paulo, Brasil). Para a determinação da estatura foi utilizada uma fita métrica milimetrada com precisão de $0,1 \mathrm{~cm}$, sendo a mesma fixada na parede com ponto zero no nível do solo. O IMC foi calculado considerando a razão entre a massa corporal e o quadrado da estatura $\left(\mathrm{kg} / \mathrm{m}^{2}\right)$.

A densidade corporal foi estimada a partir da equação generalizada de Petroski (12) mensuraram-se quatro dobras cutâneas (subescapular, tríceps, supra-ilíaca e panturrilha 
medial), seguindo procedimentos padronizados por Benedetti et al. (13). Foi utilizado um adipômetro com precisão de $0,1 \mathrm{~mm}$ (Cescorf®, Porto Alegre, Brasil). A partir da densidade corporal foi estimado o percentual de gordura através da equação de Siri ${ }^{(14)}$. Uma vez obtido o percentual de gordura foram calculadas a massa de gordura (MG) e a massa magra (MM), onde $M M=M C-M G$.

O traje utilizado para os testes foi o calção, modelo BM, a camiseta regata também do mesmo modelo e o tênis apropriado para a prática esportiva.

\section{Avaliação da aptidão física}

Os testes físicos de flexão na barra fixa, abdominal remador, corrida de máxima velocidade em $100 \mathrm{~m}$ e corrida na distância de $3200 \mathrm{~m}$ seguiram as normas e padronizações descritas por Brasil (1). Por intermédio do tempo obtido no teste de corrida na distância de $3200 \mathrm{~m}$ foi possível determinar indiretamente o consumo máximo de oxigênio (VO2max) aplicando a equação proposta por Ribisl \& Rachadorian ${ }^{(15)}$.

\section{Tratamento estatístico}

Para melhor apresentação dos resultados e caracterização dos sujeitos avaliados foi aplicada a estatística descritiva. Para verificar a distribuição dos dados, aplicou-se o teste de normalidade de Kolmogorov-Smirnov. Para verificar se existiam diferenças nas variáveis: IMC, MC, MM e VO2max pré e pós-treinamento utilizou-se o teste $\mathrm{t}$ de student para amostras pareadas; para determinar possíveis diferenças nas variáveis: percentual de gordura, MG, teste de flexão na barra fixa, no teste de corrida de 12 minutos, no teste de abdominal remador e no teste de máxima velocidade em $100 \mathrm{~m}$ pré e pós-treinamento foi utilizado o teste de Wilcoxon, para todas as análises foi adotado um nível de significância de $5 \%$.

\section{RESULTADOS}

As comparações dos efeitos do treinamento sobre a composição corporal são mostradas na TABELA 1. Os resultados deste estudo revelam que entre os índices antrométricos investigados, somente o IMC apresentou um aumento significativo após o período de treinamento.
TABELA 1

EFEITOS DO TREINAMENTO SOBRE A COMPOSIÇÃO CORPORAL DE MILITARES.

\begin{tabular}{ccc}
\hline Parâmetros & \multicolumn{2}{c}{ Composição Corporal } \\
& Pré & Pós $(\Delta$ pré-pós) \\
\hline Percentual de gordura $(\%)$ & $14,05 \pm 3,58$ & $13,31 \pm 2,88(5,3 \%)$ \\
Massa Corporal $(\mathrm{kg})$ & $70,16 \pm 8,06$ & $71,51 \pm 7,87(2 \%)$ \\
Massa de Gordura $(\mathrm{kg})$ & $15,75 \pm 3,28^{*}$ & $16,39 \pm 3,39(4,1 \%)$ \\
Massa Magra $(\mathrm{kg})$ & $54,41 \pm 4,97^{*}$ & $55,18 \pm 4,73(1,4 \%)$ \\
IMC $\left(\mathrm{kg} / \mathrm{m}^{2}\right)$ & $22,23 \pm 2,25^{*}$ & $22,70 \pm 2,20(2,1 \%)$ \\
\hline
\end{tabular}

* indica a existência de diferença significativa entre o pré e o pós-teste $(p<0,05)$.

Os resultados descritos na TABELA 2, indicam uma melhora média $12 \%$ no rendimento do grupo no Teste de $3200 \mathrm{~m}(14,17 \pm 1,38 \mathrm{~min}$ pré-treinamento para $12,46 \pm 1,27$ min póstreinamento); comportamento similar foi observado no Teste de $100 \mathrm{~m}$ melhora média de 4,1 \% (14,23 $\pm 0,46$ seg pré-treinamento para 14,05 $\pm 1,20 \mathrm{seg}$ pós-treinamento). Nos testes que envolvem força ou resistência muscular foi observado incremento médio de $43,6 \%$ no número de execuções do exercício na Barra fixa $(7,54 \pm 2,71$ pré-treinamento para $10,83 \pm 2,73$ pós-treinamento); no teste de Abdominal foi identificado um acréscimo médio de $31,5 \%$ no número de execuções $(38,48 \pm 4,02$ prétreinamento para 50,62 \pm 4,47 pós-treinamento).

TABELA 2

EFEITOS DO TREINAMENTO SOBRE A APTIDÃO FÍSICA DE MILITARES.

\begin{tabular}{ccc}
\hline & \multicolumn{2}{c}{ Aptidão Física } \\
Pré & Pós ( $\Delta$ pré-pós) \\
\hline Teste $3200 \mathrm{~m}(\mathrm{~min})$ & $14,17 \pm 1,38$ * & $12,46 \pm 1,27(12 \%)$ \\
VO2max (ml/kg/min-1) & $60,24 \pm 2,99 *$ & $63,16 \pm 3(4,8 \%)$ \\
Barra Fixa & $7,54 \pm 2,71$ * & $10,83 \pm 2,73(43,6 \%)$ \\
Abdominal & $38,48 \pm 4,02 *$ & $50,62 \pm 4,47(31,5 \%)$ \\
Teste 100m (seg) & $14,23 \pm 0,46 *$ & $14,05 \pm 1,20(4,1 \%)$ \\
\hline
\end{tabular}

* indica a existência de diferença significativa entre pré e pós-teste $(p<0,05)$.

\section{DISCUSSÃO}

O principal achado deste estudo aponta um efeito positivo do treinamento físico mais evidente nos testes de aptidão física que envolve medidas de força ou resistência muscular durante o período de formação soldados do corpo de bombeiros. As 
evidências obtidas no atual estudo demonstram melhoras pronunciadas nos testes de força ou resistência muscular que parecem contribuir justamente nos componentes físicos mais requeridos na atividade profissional de bombeiros ${ }^{(3)}$.

Parece haver uma consistência entre os resultados do presente estudo, com os apresentados em outras pesquisas que englobaram grupos militares e períodos de treinamento diferenciados. Vieira et al., ${ }^{(8)}$ verificaram modificações benéficas com apenas oito semanas de treinamento físico na distância percorrida em 12 minutos, no número de flexão dos braços na barra fixa e no somatório de dobras cutâneas, em integrantes da tropa da força de Paz do exército brasileiro.

Ceriani et al., (9) aplicaram um programa de exercício contendo 10 meses de treinamento físico, em alunos do núcleo de preparação de oficiais da reserva. Os sujeitos foram divididos em dois grupos, e o grupo experimental diminuiu os níveis de gordura e aumentou a massa magra, bem como o desempenho físico no TAF. No grupo controle a composição corporal teve comportamento inverso, e ocorreu diminuição de flexibilidade durante a investigação.

A combinação dos métodos de treinamento empregado no atual estudo pode ter contribuído para tendência de queda no percentual de gordura $(p=0,061)$ e aumento da massa corporal $(p=0,056)$ influenciada tanto pelo incremento significativo da massa magra $(p=0,035)$ quanto pela elevação da massa de gordura $(p=0,035)$. Isso também resultou numaumento significativo do IMC $(p=0,048)$ e permite inferir que a falta do controle nutricional durante o período de treinamento pode ter acarretado um balanço energético positivo, parcialmente benéfico para o ganho de massa muscular.

Contudo Oliveira e Anjos ${ }^{(16)}$ demonstraram que a pratica de exercícios físicos provoca modificações no perímetro da cintura e na gordura visceral independente de modificações no IMC, assim pode-se especular que a treinamento físico poderia acarretar em diminuição na obesidade abdominal, com ou sem modificações no IMC.

A exigência imposta pelo meio militar para o ingresso na carreira de bombeiro pode ser uma das justificativas para que os indicadores antropométricos estudados exibirem valores considerados adequados. Desta forma, o percentual de gordura pré-treinamento $(14,05 \pm$
$3,58 \% \mathrm{G})$ e pós-treinamento $(13,31 \pm 2,88 \% \mathrm{G})$, em ambas as situações apresentava-se dentro de uma classificação adequada à saúde (11 - $16 \%$ G) (25) ao considerar adultos jovens do sexo masculino. $\mathrm{O}$ mesmo ocorrer com os valores de IMC mensurados pré $\left(22,23 \pm 2,25 \mathrm{~kg} / \mathrm{m}^{2}\right)$ e pós-treinamento $(22,70$ $\pm 2,20 \mathrm{~kg} / \mathrm{m}^{2}$ ) encontram-se dentro dos valores preconizados como adequados (18,5 e $25 \mathrm{~kg} / \mathrm{m}^{2}$ ) pela Organização Mundial da Saúde (17).

Assim o presente estudo demonstra que mesmo em um grupo de sujeitos no qual os indicadores antropométricos (IMC e \%G) apresentam-se dentro de uma zona considerada saudável, esses mesmos parâmetros podem sofrer alterações favoráveis decorrentes de 12 semanas de treinamento físico.

Nos testes de aptidão neuromuscular foram encontradas melhoras nos efeitos do programa de treinamento físico de $43,6 \%$ e $31,5 \%$ para a barra fixa e abdominal, respectivamente. Dentre os principais mecanismos fisiológicos responsáveis pelos resultados obtidos nesta investigação podese destacar a modulação neural que deve ter ocorrido em decorrência do período de treinamento realizado. Neste sentido, o incremento do número de repetições observado nos exercícios de abdominais e barra fixa poderia ao menos em parte ser justificado devido a ganhos de força promovidos por possíveis fatores neurais ${ }^{(18)}$.

De fato períodos de treinamento com seis a dez semanas parecem promover uma marcante adaptação neural, sendo necessários períodos de treinamentos mais elevados para ganhos expressivos de massa muscular. Desta forma, entre os prováveis mecanismos responsáveis pelo aumento de força pode ser destacado: recrutamento de unidades motoras adicionais para maior produção de força; redução na inibição autogênica permitindo maior produção de força; redução da coativação de músculos agonistas e antagonistas; alterações nas razões de descarga de unidades motoras; alterações na junção neuromuscular ${ }^{(19)}$.

Ainda seguindo esta mesma linha de raciocínio referente a modulação neural, Fronchetti et al., (20) argumentam que a elevação do estado de condicionamento aeróbio do indivíduo tende a contribuir com o incremento do tônus vagal, principalmente em repouso. Esse aumento da atividade parassimpática induzida pelo treinamento pode diminuir o risco para desenvolvimento de doenças cardiovasculares ${ }^{(20)}$. Essa maior 
cardioproteção (maior atividade parassimpática) adquirida em virtude do treinamento físico poderia contribuir atenuando a reatividade do sistema cardiovascular quando submetido a situações de estresse psicológico ${ }^{(21)}$.

O programa de treinamento físico conduzido no presente estudo continha um predomínio do componente aeróbio e visava ao menos à manutenção dessa aptidão. Todavia ficou evidenciada um aumento significativo no $\mathrm{VO}_{2 \max }$. Já está bem estabelecido na literatura que indivíduos com maior nível de aptidão aeróbia, quando se exercitando em uma mesma carga relativa de esforço tornam-se mais eficientes e econômicos do ponto de vista energético, desta forma é possível realizar um mesmo grau de esforço com menor desgaste, poupando energia, e restabelecendo o organismo mais brevemente para a execução de uma tarefa adicional ${ }^{(22,23)}$.

Outro aspecto que pode justificar a melhora de rendimento nos testes de velocidade, refere-se à tendência na diminuição do percentual de gordura, o que pode ter contribuído para um incremento da eficiência durante as provas de corrida, isso seria atribuído a diminuição do custo de energia para uma intensidade de esforço, ou seja, maior economia de corrida, como conseqüência há uma redução no tempo de prova. De fato, Kenney \& Hodgson (24) estudando as variáveis que predizem o desempenho em corredores de elite $(5000 \mathrm{~m}$ e $3000 \mathrm{~m}$ com barreiras), concluíram que um baixo percentual de gordura está entre os importantes aspectos atribuídos ao sucesso na performance destes corredores.

A manipulação da relação volume e intensidade de treinamento podem gerar diferentes estímulos de treino, entretanto, é importante que o organismo seja submetido a uma sobrecarga mínima capaz de provocar novas adaptações, estas por sua vez devem ser adequadas ao princípio da progressão e continuidade. Deste modo, ficou demonstrado que a realização de um programa estruturado de exercícios físicos executados três vezes por semana durante 12 semanas promove modificações positivas na composição corporal e aptidão física de jovens bombeiros.

Todavia no atual estudo não foram controlados os níveis de atividade física realizada além do treinamento físico estruturado nesta investigação, da mesma forma não foi conduzido nenhum tipo de controle nutricional sobre o grupo estudado. Essas variáveis podem intervir nos resultados encontrados, assim novos estudos devem ser realizados objetivando minimizar esses possíveis efeitos, além de estudarem a efetividade de outros modelos de treinamento físico.

\section{CONCLUSÃO}

Os resultados do presente estudo permitem concluir que mesmo em um grupo de sujeitos considerados aptos no TAF, o treinamento físico realizado três vezes por semana durante um período de 12 semanas, é capaz de proporcionar melhorias na composição corporal e aptidão física.

Todos os testes físicos que englobam o TAF apresentaram melhoras significativas com 0 treinamento realizado. Assim, o treinamento físico periodizado parece induzir maiores modificações nos componentes neuromusculares e fisiológicos do que propriamente sobre a composição corporal.

A realização de estudos futuros, monitorando e orientando os hábitos alimentares em militares deve ser estimulada, de modo a demonstrar outros benefícios na composição corporal e saúde do militar. Além disso, os efeitos do treinamento demonstrado no atual estudo são proporcionais ao período de treinamento, portanto, estudos futuros devem buscar investigar e melhor demonstrar a relação entre período de treinamento e possível efeito sobre a performance no TAF.

\section{REFERÊNCIAS BIBLIOGRÁFICAS}

1. BRASIL. Estado Maior do Exército. Manual da Campanha C20-20, Treinamento Físico Militar e sua avaliação. 3ed. Brasília: EGGCF, 2002.

2. Velho, NM. Análise da aptidão física dos policiais militares do estado de Santa Catarina. (Dissertação de Mestrado). Mestrado em Educação Física. Universidade Federal de Santa Maria. Santa Maria, RS. 1994.

3. Rhea MR, Alvar BA, Gray R. Physical fitness and job performance of firefighters. J. Strength Cond Res, 2004; 18(2):348-52.

4. Duarte AFA, Pitanga Filho MV, Moraes JM, Ribeiro, LCS. Condicionamento físico aeróbio e reações psicofisiológicas a um teste de estresse. Rev Educ Física (RJ) 2003; 127:4-9. 
5. Tomasi LF. The new 1998 Army Physical Fitness Test (APFT). Standard. Soldiers 1998; (2):6-8.

6. O'connor JS, Bahrkem MS, Tetu RG. Active Army Physical Fitness Survey. Mil Med 1990; 155(12):57985.

7. Oliveira, EAM. Validade do teste de aptidão física do exército brasileiro como instrumento para a determinação das valências necessárias ao militar. Rev Educ Física (RJ) 2005; 131:30-7.

8. Vieira G, Duarte D, Silva R, Fraga C, Oliveira M, Rocha R. Efeitos de oito semanas de Treinamento Físico Militar sobre o desempenho físico, variáveis cardiovasculares e somatório de dobras cutâneas de militares de força de paz do Exército Brasileiro. Rev Educ Física (RJ) 2006; 134:30-40.

9. Cearini RB, Pontes LM, Cardoso AB, Gomes ALM, Dantas EHM. Impacto do treinamento físico militar sobre os níveis de aptidão física de alunos do núcleo de preparação de oficiais da reserva (NPOR). Rev AMRIGS. 2008; 52(3):164-69.

10. ACSM'S Guidelines for exercise testing and prescription. Lippincott, Williams \& Wilkins; 2000.

11. Borg G. Borg's perceived exertion and pain scales. Champaign, IL: Human Kinetics; 1998.

12. Petroski, EL. Equações antropométricas: subsídios para uso no estudo da composição corporal. In: Petroski EL, editor. Antropometria: técnicas e padronizações. 3 ed. Blumenau: Nova Letra, 2007.

13. Benedetti TRB, Pinho RA, Ramos VM. Dobras cutâneas. In: Petroski EL, editor. Antropometria: técnicas e padronizações. 3ed. Blumenau: Nova Letra, 2007.

14. Siri WE. Body composition from fluid space and density. In Brozek J, Hanschel A. (Eds.). Techniques for measuring body composition. Washington, D.C. National Academy of Science. 1961.

15. RibisI PM, Kachadorian WA. Maximal oxygen intake prediction in young and middle-aged males. J Sports Med Phys Fitness1969; 9(1):17-22.

16. Oliveira EAM, Anjos LA. medidas antropométricas segundo aptidão cardiorespiratória em militares da ativa, Brasil. Rev Saúde Publica 2008; 42(2):217-23.
17. World Health Organization. Physical Status: the use and interpretation of anthropometry. WHO Technical Report Series $n^{\circ}$ 854. Geneva, Switzerland: WHO, 1995.

18. Staron RS, Karapondo DL, Kraemer WJ, Fry AC, Gordon SE, Falkel JE, Hagerman FC, Hikida RS. Skeletal muscle adaptations during early phase of heavy-resistance training in men and women. J Appl Physiol 1994; 76:1247-55.

19.- Kraemer WJ, Fleck S, Evans WJ. Strength and power training: physiological mechanisms of adaptation. Exerc Sport Sci Rev 1996; 24:363-98.

20. Fronchetti L, Aguiar CA, Aguiar AF, Nakamura FY, De-oliveira, FR. Modificações da Variabilidade da Freqüência Cardíaca Frente ao Exercício e Treinamento Físico. Rev Mineira Educ Física 2007; 2:101-29.

21. Spalding TW, Jeffers LS, Porges SW, Hatfield BD. Vagal and cardiac reactivity to psychological stressors in trained and untrained men. Med Sci Sports Exerc 2000; 32(3):581-91.

22. Ästrand PO, Rodahl K. Textbook of work physiology. New York: McGraw-Hill; 1986.

23. Denadai BS. Índices Fisiológicos de Avaliação Aeróbia: Conceitos e Aplicações. Ribeirão Preto: B.S.D.; 1999.

24. Kenney WL, Hodgdon JL. Variables predictive of performance in elite middle-distance runners. $\mathrm{Br} \mathrm{J}$ Sports Med 1985; 19(4):207-09.

25. Lohman TG. Advances in body composition assessment. Champaign, IL: Human Kinetics. 1992.

Física (RJ) 2003; 127:4-9.

5. Tomasi LF. The new 1998 Army Physical Fitness Test (APFT). Standard. Soldiers 1998; (2):6-8.

6. O'connor JS, Bahrkem MS, Tetu RG. Active Army Physical Fitness Survey. Mil Med 1990; 155(12):579-85.

7. Oliveira, EAM. Validade do teste de aptidão física do exército brasileiro como instrumento para a determinação das valências necessárias ao militar. Rev Educ Física (RJ) 2005; 131:30-7.

8. Vieira G, Duarte D, Silva R, Fraga C, Oliveira M, Rocha R. Efeitos de oito semanas de Treinamento 
Físico Militar sobre o desempenho físico, variáveis cardiovasculares e somatório de dobras cutâneas de militares de força de paz do Exército Brasileiro. Rev Educ Física (RJ) 2006; 134:30-40.

9. Cearini RB, Pontes LM, Cardoso AB, Gomes ALM, Dantas EHM. Impacto do treinamento físico militar sobre os níveis de aptidão física de alunos do núcleo de preparação de oficiais da reserva (NPOR). Rev AMRIGS. 2008; 52(3):164-69.

10. ACSM'S Guidelines for exercise testing and prescription. Lippincott, Williams \& Wilkins; 2000.

11. Borg G. Borg's perceived exertion and pain scales. Champaign, IL: Human Kinetics; 1998.

12. Petroski, EL. Equações antropométricas: subsídios para uso no estudo da composição corporal. In: Petroski $E L$, editor. Antropometria: técnicas e padronizações. 3 ed. Blumenau: Nova Letra, 2007.

13. Benedetti TRB, Pinho RA, Ramos VM. Dobras cutâneas. In: Petroski EL, editor. Antropometria: técnicas e padronizações. 3ed. Blumenau: Nova Letra, 2007.

14. Siri WE. Body composition from fluid space and density. In Brozek J, Hanschel A. (Eds.). Techniques for measuring body composition. Washington, D.C. National Academy of Science. 1961.

15. RibisI PM, Kachadorian WA. Maximal oxygen intake prediction in young and middle-aged males. J Sports Med Phys Fitness1969; 9(1):17-22.

16. Oliveira EAM, Anjos LA. medidas antropométricas segundo aptidão cardiorespiratória em militares da ativa, Brasil. Rev Saúde Publica 2008; 42(2):217-23.

17. World Health Organization. Physical Status: the use and interpretation of anthropometry. WHO Technical Report Series $n^{\circ}$ 854. Geneva, Switzerland: WHO, 1995.

18. Staron RS, Karapondo DL, Kraemer WJ, Fry AC, Gordon SE, Falkel JE, Hagerman FC, Hikida RS. Skeletal muscle adaptations during early phase of heavy-resistance training in men and women. J Appl Physiol 1994; 76:1247-55.
19.- Kraemer WJ, Fleck S, Evans WJ. Strength and power training: physiological mechanisms of adaptation. Exerc Sport Sci Rev 1996; 24:363-98.

20. Fronchetti L, Aguiar CA, Aguiar AF, Nakamura FY, De-oliveira, FR. Modificações da Variabilidade da Freqüência Cardíaca Frente ao Exercício e Treinamento Físico. Rev Mineira Educ Física 2007; 2:101-29.

21. Spalding TW, Jeffers LS, Porges SW, Hatfield BD. Vagal and cardiac reactivity to psychological stressors in trained and untrained men. Med Sci Sports Exerc 2000; 32(3):581-91.

22. Ästrand PO, Rodahl K. Textbook of work physiology. New York: McGraw-Hill; 1986.

23. Denadai BS. Índices Fisiológicos de Avaliação Aeróbia: Conceitos e Aplicações. Ribeirão Preto: B.S.D.; 1999.

24. Kenney WL, Hodgdon JL. Variables predictive of performance in elite middle-distance runners. $\mathrm{Br} \mathrm{J}$ Sports Med 1985; 19(4):207-09.

25. Lohman TG. Advances in body composition assessment. Champaign, IL: Human Kinetics. 1992.

4. Silva Dantas PM, Alonso L. Futsal e Dermatoglifia. In: Dantas EHM, Fernandes Filho J, editors. Atividade física em ciências da saúde. 1 ed. Rio de Janeiro: editora Shape, 2005.

Correspondência:

Edio Luiz Petroski

Universidade Federal de Santa Catarina

Centro de Desportos

Campus Universitário - Trindade - Caixa postal 476

CEP - 88040-900 Florianópolis, SC. Brasil

E-mail: petroski@cds.ufsc.br 